Document downloaded from:

http://hdl.handle.net/10251/120633

This paper must be cited as:

Andreu-Estellés, C.; Garcia-Pardo, C.; Castelló-Palacios, S.; Vallés Lluch, A.; Cardona Marcet, N. (2018). Frequency Dependence of UWB In-Body Radio Channel Characteristics. IEEE Microwave and Wireless Components Letters. 28(4):359-361. https://doi.org/10.1109/LMWC.2018.2808427

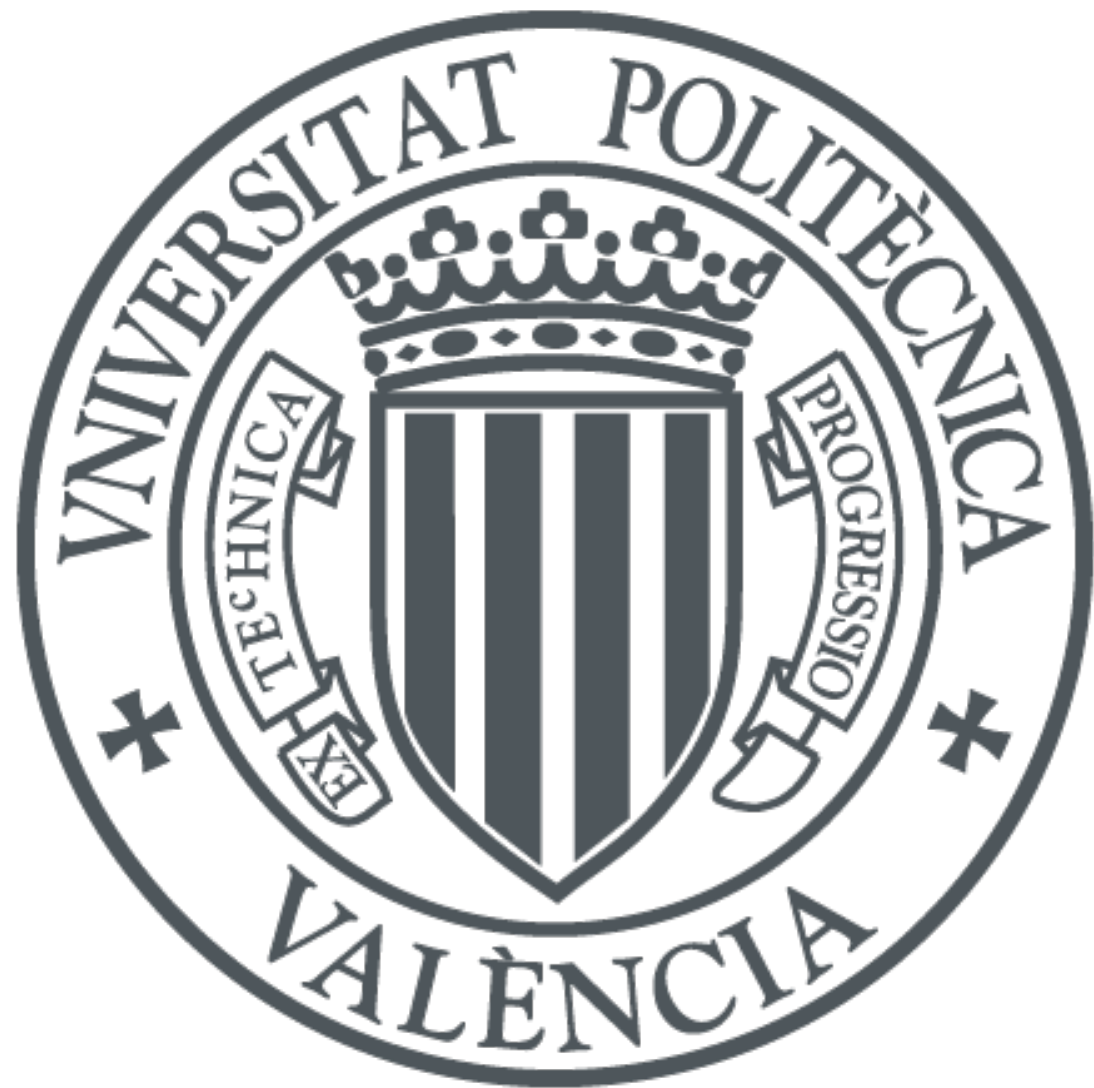

The final publication is available at

http://doi.org/10.1109/LMWC.2018.2808427

Copyright Institute of Electrical and Electronics Engineers

Additional Information

(c) 2018 IEEE. Personal use of this material is permitted. Permission from IEEE must be obtained for all other users, including reprinting/ republishing this material for advertising or promotional purposes, creating new collective works for resale or redistribution to servers or lists, or reuse of any copyrighted components of this work in other works. 


\title{
Frequency Dependence of UWB In-Body Radio Channel Characteristics
}

\author{
Carlos Andreu, Concepcion Garcia-Pardo, Sergio Castelló-Palacios, Ana Vallés-Lluch \\ and Narcís Cardona
}

\begin{abstract}
In this work, a research of Ultra-wideband (UWB) in-body channel by using a high accurate phantom is performed in order to evaluate the impact of frequency dependence of human tissues on the channel characteristics. Hence, a phantombased measurement campaign from 3.1 to $5.1 \mathrm{GHz}$ has been conducted. From post-processing data, the path loss is assessed considering subbands of $500 \mathrm{MHz}$ as well as the entire frequency range under test. In addition, the correlation in transmission is computed and discussed.
\end{abstract}

Index Terms - Ultra-Wideband, in-body communications, implanted sensors, channel performance, Body Area Networks.

\section{INTRODUCTION}

$\mathrm{D}$ ata rate of current wireless in-body medical devices is far from the performance of other telecommunication systems today in force. Thus, many research works have addressed the use of the first two gigahertz of the UWB band as a potential candidate for the revision of the current standard for in-body communications [1]. Nevertheless, a large number of validations should be carried out to choose this technology as the most appropriate for this purpose. To benefit from UWB systems, the in-body radio channel should be thoroughly investigated considering pulse-based schemes, which use large bandwidths, as well as multicarrier-based schemes, which split the available spectrum in subbands. UWB impulse radio (UWB-IR) and multiband orthogonal frequency-division multiplexing (MB-OFDM) are examples of these transmission schemes. Therefore, radio channel performance for the whole used bandwidth as well as for each $500 \mathrm{MHz}$ subband should be studied separately. It is relatively easy to find papers addressing the effect of frequency [2]-[4]. However, to the best of the authors' knowledge, there are no works dealing with the performance of UWB spectrum per subbands. Channel diversity can contribute to the emergence of new medical applications. UWB localization techniques, among other applications, could benefit from this fact [5]. Many studies address the diversity of off-body systems [6]. However, it is not so easy to find studies dealing with UWB in-body channel diversity and its variation as function of frequency.

This letter is devoted to the evaluation of the effect of

This work was supported by the Ministerio de Economía y Competitividad, Spain (TEC2014-60258-C2-1-R), by the European FEDER funds.

C. Andreu, C. Garcia-Pardo, S. Castelló-Palacios and N. Cardona are with iTEAM, Universitat Politècnica de València, 46022 Valencia, Spain (e-mail: \{caranes, cgpardo, sercaspa, ncardona\} @iteam.upv.es).

A. Vallés-Lluch and S. Castelló-Palacios are with the Centre for Biomaterials and Tissue Engineering, Universitat Politècnica de València, 46022 Valencia, Spain (e-mail: avalles@ ter.upv.es). frequency dependence of human tissues in the UWB in-body channel performance. For this purpose, an experimental phantom-based measurement campaign from 3.1 to $5.1 \mathrm{GHz}$ reproducing an in-body to on-body scenario has been carried out. For that, a high accurate phantom in a novel setup with a high spatial resolution positioner has been used. From the obtained results, the path loss values in multiple 3D locations within a distance between antenna centers from $2.8 \mathrm{~cm}$ to 8 $\mathrm{cm}$ have been computed. Afterwards, the most suitable path loss approximation models for each subband of $500 \mathrm{MHz}$ as well as for the whole frequency range are compared. Moreover, the correlation in transmission is discussed.

\section{MeAsurement Procedure}

\section{A. Setup}

For the in-body to on-body (IB2OB) scenario, the implanted antenna is placed inside the human body, whereas the on-body one is hung over the skin surface [1]. In this scenario, the information collected by implanted sensors is usually transmitted from in-body transmitting antennas to on-body receivers through the human body tissues. Since the propagation medium is not the free space, both in-body and on-body antennas should be carefully designed considering the human tissues. Hence, two different kinds of antennas were selected as transmitter and receiver. An UWB monopole antenna acted as the implanted transmitter, which is a suitable candidate since it was designed considering the propagation medium [2]. On the other hand, an UWB on-body slotted patch antenna, which acted as receiver, was optimized considering the tissue layers of the human abdominal region [7]. This antenna includes a reflecting plane in order to increase its directivity and thus reduce the backward radiation.

Reproducing the transmission through different body tissues in a multilayer experimental setup is very complicated. Besides, the implanted antenna should have freedom of movement to characterize the channel in several in-body locations. The different human body tissues that are located in the human thoracic and abdominal regions (skin, muscle, heart, kidney, small bowel, etc.) have a similar complex permittivity within the UWB spectrum [8]. For these reasons, most research works use a single-composition liquid phantom which aim at imitating the complex permittivity of the human muscle tissue [3], [4], [9]. However, achieving a high accurate phantom within the whole UWB range can become unrealizable. For this work, a human muscle tissue-equivalent liquid phantom was used in order to emulate the propagation from inside to outside the body [10]. As far as we know, this phantom achieves the most accurate imitation of the relative 
permittivity of this tissue within the studied frequency band [11]. Fig. 1 depicts the experimental IB2OB scenario. The phantom was poured into a polypropylene (PP) box of $30 \times 30 \times 15 \mathrm{~cm}^{3}$ which imitates a cross section of the human body torso [2]. The thickness of container's wall was considered to be negligible. The on-body antenna was directly placed over the external container's face, whereas the in-body one was submerged into the phantom.

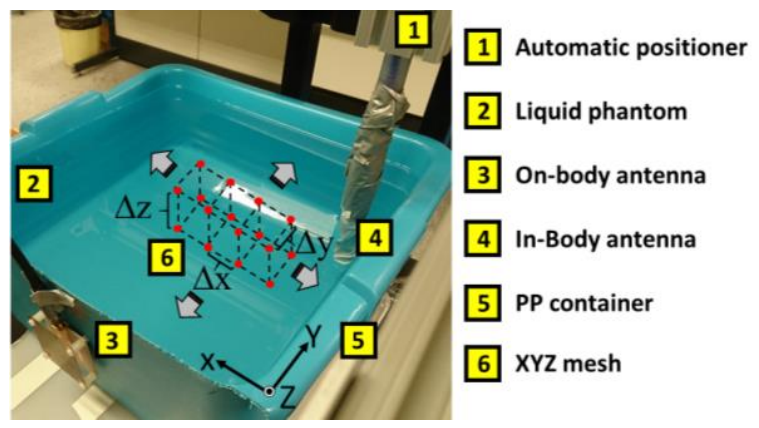

Fig. 1. Experimental IB2OB measurement setup.

The transmitting antenna was located in different spatial $(\mathrm{x}, \mathrm{y}, \mathrm{z})$ positions by using a 3 -axis positioner, whereas the onbody receiver was fixed at the center of the outer face of the box. The in-body antenna was moved in steps of $\Delta x=\Delta y=\Delta z$ $=1 \mathrm{~cm}$ along the three axes (see Fig. 1). This antenna was located in a XYZ mesh of $7 \times 7 \times 3$ measurement points. The distance between antenna centers was computed considering the absolute position of the antennas.

\section{B. Methodology}

Channel measurements $\left(S_{21}\right)$ were captured by a E5072A ENA vector network analyzer. This was calibrated through a full 2-port calibration from $3.1-5.1 \mathrm{GHz}$ with 1601 resolution points. The noise floor was at $-100 \mathrm{dBm}$. Only those $S_{21}$ samples $10 \mathrm{~dB}$ above the noise level were considered to capture mainly the direct path contribution. Both $S_{11}$ and $S_{22}$ were measured in each antenna position to ensure a proper antenna matching across the frequency band.

\section{PATH LOSS}

The path loss in each in-body antenna location was calculated as described in [2]. The path loss for each of the first four subbands at the lower part of UWB frequency band was calculated. Each subband covers a bandwidth of $500 \mathrm{MHz}$ from 3.1 to $5.1 \mathrm{GHz}$. In addition, the path loss values considering the whole frequency range from 3.1 to $5.1 \mathrm{GHz}$ were obtained. It should be taken into account that the path loss is influenced by the antenna behavior, thus being a radio link budget evaluation. However, path loss is the most used term in the literature [1], [2], [4].

According to the observed path loss values, on the one hand, they were fitted by a linear approximation model as:

$$
P L_{\text {lin }}(d B)=P L_{0}(d B)+\alpha \cdot d(\mathrm{~cm}),
$$

where $P L_{0}$ is the value of the path loss when the distance between antenna centers, $d$, tends to 0 and $\alpha$ is a fitting parameter. On the other hand, a log-distance model to approximate the path loss values was considered. The logdistance model follows the following expression:

$$
P L_{\mathrm{log}}(d B)=P L_{0, d_{r f}}(d B)+10 \gamma \log _{10}\left(d(\mathrm{~cm}) / d_{r e f}\right),
$$

being $P L_{0, d_{r e f}}$ the path loss value in a reference distance $d_{r e f}=1$ $\mathrm{cm}$, and $\gamma$ the path loss exponent.

\section{A. Discussion}

Fig. 2 shows the path loss values computed from $d=2.8 \mathrm{~cm}$ to $8 \mathrm{~cm}$ as well as the fitted curves for the IB2OB scenario described in section II.A. As can be observed, the path loss values are higher as the frequency band increases.
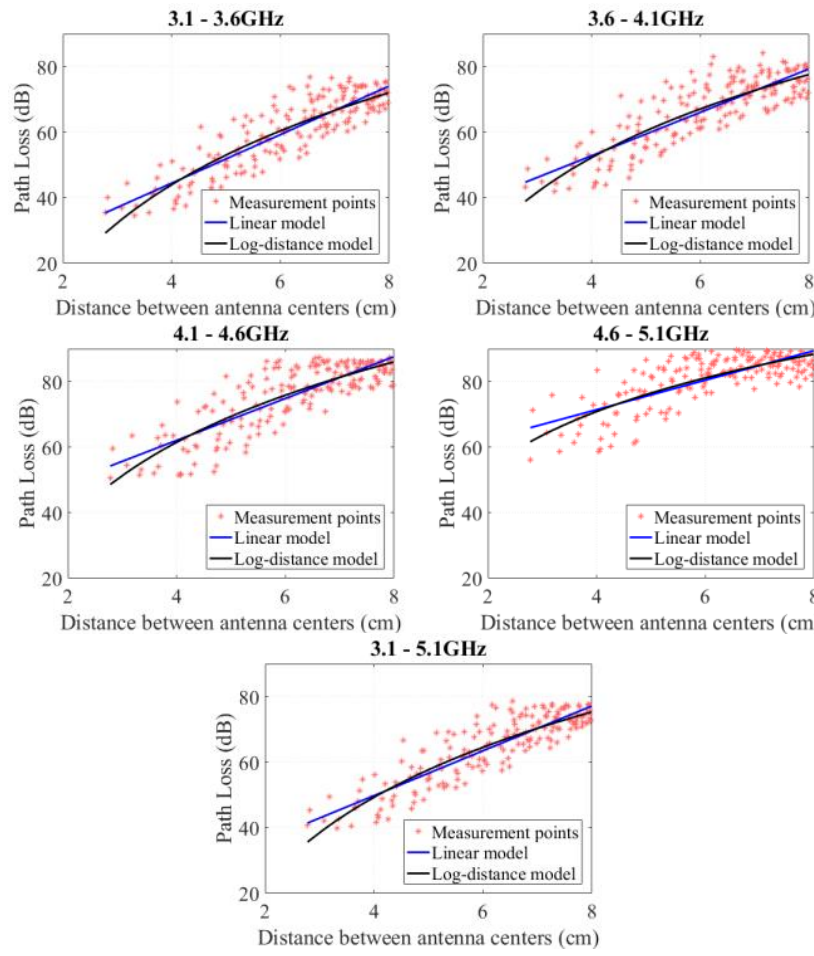

Fig. 2. Path loss as a function of distance between antenna centers

TABLE I

FITTING PARAMETERS OF THE APPROXIMATION MODELS

\begin{tabular}{|c|ccc|ccc|}
\hline \hline \multirow{2}{*}{$\begin{array}{c}\text { Frequency } \\
\text { range } \\
(\mathrm{GHz})\end{array}$} & \multicolumn{3}{|c|}{ Linear model } & \multicolumn{3}{c|}{ Log-distance model } \\
\cline { 2 - 7 } & $\mathrm{PL}_{0}$ & $\alpha$ & RMSE & $\mathrm{PL}_{0}$ & $\gamma$ & RMSE \\
\hline $3.1-3.6$ & 14.826 & 7.405 & 5.287 & -12.202 & 9.329 & 5.345 \\
$3.6-4.1$ & 26.299 & 6.627 & 5.618 & 1.482 & 8.431 & 5.531 \\
$4.1-4.6$ & 36.496 & 6.394 & 5.723 & 12.432 & 8.150 & 5.619 \\
$4.6-5.1$ & 53.434 & 4.502 & 5.215 & 35.829 & 5.820 & 5.004 \\
\hline $3.1-5.1$ & 22.406 & 6.846 & 4.843 & 2.814 & 8.656 & 4.840 \\
\hline \hline
\end{tabular}

Table I presents the path loss fitting parameters along with the RMSE for each approximation model considering each subband as well as the whole bandwidth. As can be seen, parameter $\alpha$ is lower as the subband is located in higher frequency bands for the linear approximation model, i.e., the slope of the curve is less steep. The same occurs with regard to the $\gamma$ exponent in the case of the log-distance model as the subband increases in frequency. The path loss exponent is lower in the upper subbands (see Table I). This is by the fact that losses raise the noise level at large distances, whereas they grow at short distances. Regarding $P L_{0}$, its value increases as 
the subband is higher in frequency for both approximation models. It can be observed how $P L_{0}$ is negative for the lowest frequency subband in the log-distance model. Such negative value is in concordance with a best fitting -lower RMSE- of the path loss for a linear trend. This can be observed in Table I, where the RMSE is lower for the linear than for the logdistance one. Even though the RMSE is lower for the linear, the error is practically the same by using either approximation model. According to the results of Table I, it can be also concluded that the path loss is better fitted by a linear model only in the lowest frequency subband. Nevertheless, the path loss increasingly follows a logarithmic trend as the considered frequency band is above $3.6 \mathrm{GHz}$. This can be noted since the difference between RMSE values of both models is higher as the used frequency band increases. That is, 0.087 for the second subband (3.6-4.1 GHz) and 0.212 for the fourth one (4.6-5.1 GHz), what leads to an increment of $143.67 \%$. With regard to the path loss considering the whole bandwidth, the best fitting is achieved with the log-distance model, as concluded in [2]. However, the RMSE is quite similar in both models within this frequency and distance range.

\section{CORRELATION In TRANSMisSiON}

The diversity of the channel in transmission was assessed by the correlation coefficients. The correlation coefficients between two different impulse channel responses were computed as the maximum of the correlation [2]. The impulse responses were calculated by applying an Inverse Fast Fourier Transform to the $S_{2 I}(f)$ samples. The channel impulse response in a reference location of the in-body transmitter was correlated with those located in the same $\mathrm{Z}$ plane $(\mathrm{z}=0)$ as well as at different heights $(\mathrm{z}=\Delta \mathrm{z}, \mathrm{z}=2 \Delta \mathrm{z})$ to analyze the correlation in transmission. The location of the reference impulse response was set at the center of XY plane at $\mathrm{z}=0$.

From the correlation coefficients, the probability that these coefficients are equal to or greater than 0.8 was calculated. In Table II, the probability values for each subband and for the entire frequency range are shown. For each of these values, different areas were considered. The first value takes into account the correlation coefficients from $\mathrm{Y}=1$, i.e., the closest locations between antennas in $\mathrm{Y}$ axis, to $\mathrm{Y}=4\left(1^{\text {st }}\right.$ half), where the reference position is located, and all antenna locations in $\mathrm{X}$ axis. The second one, considers positions from $\mathrm{Y}=4$ to $\mathrm{Y}=7$ ( $2^{\text {nd }}$ half $)$ and all antenna locations in $\mathrm{X}$ axis as well. Finally, the last value assumes all antenna locations in the same XY plane. From Table II, one can observe that the correlation is getting lower as the distance between in-body transmitters increases in $\mathrm{Z}$ axis for all the cases, as concluded in [2]. Besides, the correlation is lower for those locations farther in $\mathrm{Y}$ axis. This decrement is influenced by multipath effect due to the multiple bounces on the walls of the box. The variations in $\mathrm{z}=0$ can be caused by the unpredictable multipath effect. Moreover, the correlation per subband is higher when the subband is located at upper frequencies whatever the distance between transmitters in $\mathrm{Z}$ axis. Regarding the correlation considering the whole frequency band, it decreases as the distance between antennas increases in height as well. Besides, the correlation values considering the entire bandwidth are close to those obtained at the lower subbands for $\mathrm{z} \leq \Delta \mathrm{z}$. However, the correlation in the lowest subband is quite lower than the rest of cases at $\mathrm{z}=2 \Delta \mathrm{z}$.

TABLE II

PROBABILITY FOR CORRELATION COEFFICIENTS $>0.8$

\begin{tabular}{cccc}
\hline \hline $\begin{array}{c}\text { Frequency } \\
\text { range } \\
(\mathrm{GHz})\end{array}$ & $\begin{array}{c}\mathbf{z = 0} \\
1^{\mathrm{st}} \mathrm{Half} / 2^{\text {nd }} \text { Half/ } \\
\text { Whole }\end{array}$ & $\begin{array}{c}\mathbf{z}=\mathbf{\Delta} \\
1^{\mathrm{st}} \mathrm{Half} / 2^{\text {nd }} \text { Half/ } \\
\text { Whole }\end{array}$ & $\begin{array}{c}\mathbf{z}=\mathbf{2 \Delta \mathbf { z }} \\
1^{\mathrm{st}} \mathrm{Half} / 2^{\text {nd }} \mathrm{Half} / \\
\text { Whole }\end{array}$ \\
\hline $3.1-3.6$ & $0.667 / 0.286 / 0.531$ & $0.524 / 0.191 / 0.469$ & $0.381 / 0.143 / 0.347$ \\
$3.6-4.1$ & $0.714 / 0.191 / 0.551$ & $0.714 / 0.333 / 0.510$ & $0.571 / 0.191 / 0.429$ \\
$4.1-4.6$ & $0.809 / 0.238 / 0.592$ & $0.714 / 0.333 / 0.571$ & $0.619 / 0.333 / 0.531$ \\
$4.6-5.1$ & $0.857 / 0.667 / 0.796$ & $0.857 / 0.619 / 0.755$ & $0.762 / 0.524 / 0.694$ \\
\hline $3.1-5.1$ & $0.667 / 0.238 / 0.512$ & $0.667 / 0.191 / 0.490$ & $0.619 / 0.095 / 0.429$ \\
\hline \hline
\end{tabular}

V. CONCLUSIONS

The path loss for an IB2OB scenario was shown to be better fitted by a log-distance model considering $500 \mathrm{MHz}$ subbands as well as $3.1-5.1 \mathrm{GHz}$ frequency range from 2.8 to 8 $\mathrm{cm}$. Moreover, the correlation decreases as the distance between transmitters increases in height. Furthermore, the correlation in upper frequency subbands is higher than that of the low frequency ones. The results also evidence that the correlation values for the whole bandwidth are quite similar to those obtained at the lower subbands.

\section{REFERENCES}

[1] R. Chavez-Santiago et al., "Propagation models for IEEE 802.15.6 standardization of implant communication in body area networks," IEEE Commun. Mag., vol. 51, no. 8, pp. 80-87, Aug. 2013.

[2] C. Andreu, S. Castelló-Palacios, C. Garcia-Pardo, A. Fornes-Leal, A. Vallés-Lluch, and N. Cardona, "Spatial In-Body Channel Characterization Using an Accurate UWB Phantom," IEEE Trans. Microw. Theory Tech., vol. 64, no. 11, pp. 3995-4002, Nov. 2016.

[3] R. Chavez-Santiago, C. Garcia-Pardo, A. Fornes-Leal, A. Valles-Lluch, I. Balasingham, and N. Cardona, "Ultra wideband propagation for future in-body sensor networks," in 2014 IEEE 25th Annual International Symposium on Personal, Indoor, and Mobile Radio Communication (PIMRC), 2014, pp. 2160-2163.

[4] D. Anzai et al., "Experimental Evaluation of Implant UWB-IR Transmission With Living Animal for Body Area Networks," IEEE Trans. Microw. Theory Tech., vol. 62, no. 1, pp. 183-192, Jan. 2014.

[5] S. Gezici, G. B. Giannakis, H. Kobayashi, A. F. Molisch, H. V. Poor, and Z. Sahinoglu, "Localization via ultra-wideband radios: a look at positioning aspects for future sensor networks," IEEE Signal Process. Mag., vol. 22, no. 4, pp. 70-84, Jul. 2005.

[6] M. Marinova et al., "Diversity Performance of Off-Body MB-OFDM UWB-MIMO," IEEE Trans. Antennas Propag., vol. 63, no. 7, pp. 31873197, Jul. 2015.

[7] E. Miralles, C. Andreu, M. Cabedo-Fabrés, M. Ferrando-Bataller, and J. F. Monserrat, "UWB on-body slotted patch antennas for in-body communications," in 2017 11th European Conference on Antennas and Propagation (EUCAP), 2017, pp. 167-171.

[8] C. Gabriel, "Compilation of the Dielectric Properties of Body Tissues at RF and Microwave Frequencies.," Brooks Air Force, N.AL/OE-TR1996-0037, San Antonio, TX, 1996.

[9] C. Andreu, C. Garcia-Pardo, A. Fornes-Leal, M. Cabedo-Fabrés, and N. Cardona, "UWB in-body channel performance by using a direct antenna designing procedure," in 2017 11th European Conference on Antennas and Propagation (EUCAP), 2017, pp. 278-282.

[10] S. Castelló-Palacios, C. Garcia-Pardo, A. Fornes-Leal, N. Cardona, and A. Vallés-Lluch, "Tailor-Made Tissue Phantoms Based on Acetonitrile Solutions for Microwave Applications up to $18 \mathrm{GHz}$," IEEE Trans. Microw. Theory Tech., vol. 64, no. 11, pp. 3987-3994, Nov. 2016.

[11] N. Cardona Marcet, S. Castelló Palacios, A. Fornés Leal, C. García Pardo, and A. Vallés Lluch, "Synthetic Model of Biological Tissues for Evaluating the Wireless Transmission of Electromagnetic Waves," [Spanish Patent], PCT/ES2016/070912, 06/29/2017. 